Chapman University

Chapman University Digital Commons

Psychology Faculty Articles and Research

Psychology

$11-3-2015$

\title{
Effects of Competing News Media Frames of Weight on Antifat Stigma, Beliefs about Weight, and Support for Obesity-Related Public Policies
}

David Frederick

Chapman University, dfrederi@chapman.edu

Abigail C. Saguy

University of California, Los Angeles

Gaganjyot Sandhu

Chapman University

Traci Mann

University of Minnesota

Follow this and additional works at: http://digitalcommons.chapman.edu/psychology_articles

\section{Recommended Citation}

Frederick, D. A., Saguy, A. C., Sandhu, G. \& Mann, T. Effects of competing news media frames of weight on antifat stigma, beliefs about weight and support for obesity-related public policies. Int J Obes 40, 543-549 (2016).

doi: $10.1038 /$ ijo.2015.195 


\section{Effects of Competing News Media Frames of Weight on Antifat Stigma, Beliefs about Weight, and Support for Obesity-Related Public Policies}

\section{Comments}

This is a pre-copy-editing, author-produced PDF of an article accepted for publication in International Journal of Obesity, volume 40, in 2016 following peer review. The definitive publisher-authenticated version is available online at DOI: 10.1038/ijo.2015.195

\section{Copyright}

Macmillan Publishers Limited 


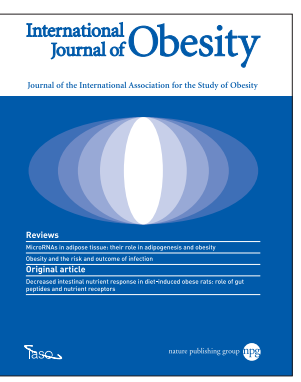

Effects of competing news media frames of weight on antifat stigma, beliefs about weight, and support for obesity-related public policies

D A Frederick, A C Saguy, G Sandhu, T Mann

Cite this article as: D A Frederick, A C Saguy, G Sandhu, T Mann, Effects of competing news media frames of weight on antifat stigma, beliefs about weight, and support for obesity-related public policies, International Journal of Obesity accepted article preview 23 September 2015; doi: 10.1038/ijo.2015.195.

This is a PDF file of an unedited peer-reviewed manuscript that has been accepted for publication. NPG are providing this early version of the manuscript as a service to our customers. The manuscript will undergo copyediting, typesetting and a proof review before it is published in its final form. Please note that during the production process errors may be discovered which could affect the content, and all legal disclaimers apply.

Received 19 May 2015; revised 22 August 2015; accepted 30 August 2015; Accepted article preview online 23 September 2015 
Effects of Competing News Media Frames of Weight

on Antifat Stigma, Beliefs About Weight, and Support for Obesity-Related Public Policies

David A. Frederick

Crean School of Health and Life Sciences, Chapman University

Abigail C. Saguy

Department of Sociology, UCLA

Gaganjyot Sandhu

Crean School of Health and Life Sciences, Chapman University

Traci Mann

Department of Psychology, University of Minnesota

Correspondence to: David Frederick at Crean School of Health and Life Sciences, Chapman

University; One University Drive; Orange, CA 92866. Enderflies1 @ aol.com or Abigail Saguy, saguy@ soc.ucla.edu

Word count: $=3997(\operatorname{Max}=4000)$

Abstract count: $289(\operatorname{Max}=300)$

Reference count: $41(\mathrm{Max}=60)$

Table/Figure count: $4(\mathrm{Max}=6)$

There are no competing financial interests or conflicts of interest to disclose. 


\begin{abstract}
Objectives: In the popular news media, public health officials routinely emphasize the health risks of obesity and portray weight as under personal control. These messages may increase support for policies designed to reduce rates of obesity, but can also increase antifat stigma. Less often, the media cover "Health at Every Size" or "Fat Rights" perspectives, which may have the opposite effects. We investigated how exposure to different "fat frames" shifts attitudes about weight and support for obesity policies. Methods: Across four experiments $(N=2187)$, people read constructed news articles framing fatness as negative (unhealthy, controllable, acceptable to stigmatize) or positive (healthy, uncontrollable, unacceptable to stigmatize). Results: Compared to people who read fat-positive frames, people who read fat-negative frames expressed more: belief in the health risks of being fat $(d \mathrm{~s}=0.95-1.22)$, belief weight is controllable $(d \mathrm{~s}=0.38$ $0.55)$, support for charging obese people more for health insurance $(d s=0.26-0.77)$, antifat prejudice (in three out of four experiments, $d \mathrm{~s}=0.28-0.39$ ), willingness to discriminate against fat people $(d s=0.39-0.71)$, and less willingness to celebrate body-size diversity $(d s=0.37-0.64)$. They were also less willing to say that women at the lower end of the obese range could be healthy at their weights. Effects on support for public policies, however, were generally small and/or non-significant. Compared to a control condition, exposure to fat-positive frames generally shifted attitudes more than fat-negative frames. In Experiment 4, adding a message about the unacceptability of weight-based discrimination to unhealthy/controllable news articles only reduced antifat stigma on one of three measures compared to articles adding a discrimination-acceptable message. Conclusions: Exposure to different news frames of fat can shift beliefs about weight-related health risks and weight-based stigma. Shifting policy attitudes, however, is more challenging.
\end{abstract}

Keywords: obesity; prejudice; public health; body image; weight stigma; antifat attitudes; frame; public policy; news media 


\section{Introduction}

Public health officials consider obesity a leading public health crisis and encourage people to lose or control their weight. The popular news media disseminates this view widely, especially in reports on medical studies (ref. 1, 2, 3). The Justification-Suppression model of prejudice (ref. 4) suggests that people are more likely to express prejudice, and feel justified in doing so, when they perceive a trait to be both negative (e.g., unhealthy) and under personal control. Consistent with this model, people who believe weight is under personal control are more likely to express negative attitudes toward heavier men and women (ref. 5, 6). Based on this model, news reports asserting that weight is controllable and that high body fat levels are unhealthy could lead to increased antifat stigma. Antifat stigma, in turn, leads to increased stress, impaired health, and reduced willingness to seek medical treatment (ref. 7, 8, 9).

Some media reports, however, do not portray fatness as harmful, controllable, or acceptable to stigmatize. In this paper, we use experimental methods to measure how exposure to different representations of fatness in popular news media reports affect beliefs about the health risks of fatness, support for different obesity-related public policies, and acceptance or rejection of antifat stigma.

\section{Framing the Problem of Fatness}

There are several distinct negative ways news reports frame fatness. The public health crisis frame presents obesity as a public health crisis warranting government intervention, as when former U.S. Surgeon General Richard Carmona said that its death toll "will dwarf 9-11 or any other terrorist attempt" (ref. 10). The personal responsibility frame blames weight gain on bad food and exercise choices and presents willpower and self-control as crucial for weight loss (ref. 2, 3, 11). The weight discrimination as justified (henceforth discrimination-justified) frame 
suggests that fatness is an acceptable target of prejudice. Content analyses of online news media images and videos show that the news media routinely portray fat men and women in stigmatizing ways (ref. 12, 13).

There are two fat-positive news media frames. Some researchers, clinicians, and activists promote a Health at Every Size (HAES) frame (ref. 14). According to this perspective, weight loss attempts typically produce neither weight loss nor improved health (ref. 15) and even when elevated Body Mass Index (BMI) is associated with mortality or morbidity, it is not the cause. Rather, poor nutrition, sedentary lifestyle, poverty, weight-based stigma, or other factors cause both negative health outcomes and weight gain (ref. 16). This frame suggests that public officials should emphasize healthy eating and exercise rather than weight loss. Some public health officials have cautioned that disseminating research consistent with a HAES perspective could erode support for anti-obesity policies (ref. 17, 18), but it is unclear if this concern is empirically supported.

The fat rights frame goes further than HAES, presenting fatness as a form of diversity and denouncing weight-based discrimination (ref. 19, 20, 21, 22). Fat rights activists - who reject the medical terms "overweight" and "obesity" and reclaim "fat" and "fatness" as valueneutral - promote this perspective in books, blogs and through organizations such as the National Association to Advance Fat Acceptance. They argue that news media reporting on the "obesity epidemic" increases weight-based prejudice and stigma (ref. 23).

\section{Past Experimental Research on Frames}

The results of previous experimental studies attempting to manipulate antifat attitudes are inconsistent (ref. 24). Of the 16 articles reviewed by Daníelsdóttira and colleagues (ref. 24) most 
$(N=13)$ included only a single experiment, raising questions about replicability with a given set of stimuli and outcome measures.

More recently, in 4 out of 5 experiments, exposure to real news articles presenting the public health crisis frame increased expressions of antifat attitudes (ref. 25). People exposed to stigmatizing photos of obese men and women in the media reported more antifat attitudes than people exposed to more positive representations (ref. 26, 27). When exposed to scientific evidence that weight discrimination is a common problem in the workplace, women, but not men, became more willing to support anti-discrimination laws (ref. 28).

Negative representations of obese people can also impact people's willingness to deny medical treatments to obese women (ref. 29) and to charge them more for health insurance (ref. 25). Other research has found, however, that exposure to framings of obesity had limited or no effects on policy attitudes (ref. 30).

\section{Purpose of Studies}

These studies seek to clarify inconsistencies in the literature on the effects of different framings of obesity. We examined the effects of exposure to Fat-Negative versus Fat-Positive frames across multiple experiments. To see how the different components of these frames affect attitudes toward fatness, we created eight news articles that systematically varied by whether they presented fatness as: 1) healthy vs. unhealthy; 2) controllable vs. uncontrollable; and 3) discrimination as justified vs. unacceptable.

\section{Hypotheses}

Hypothesis 1: Compared to people exposed to Fat-Positive frames, people exposed to Fat-Negative frames will report stronger beliefs that fatness is unhealthy, that weight is under 
personal control, more antifat stigma (Experiments 1-4), and more support for obesity-reducing public policies (Experiments 1-3).

Hypothesis 2. Compared to a control condition, Fat-Negative frames will increase whereas Fat-Positive frames will reduce — beliefs that fatness is unhealthy, antifat stigma, and support for obesity-reducing policies (Experiment 3).

Hypothesis 3. There will be main effects of exposure to articles presenting fatness as unhealthy versus healthy, fatness as controllable versus uncontrollable, and weight-based discrimination as justified vs. unacceptable. People exposed to messages that fatness is unhealthy or controllable or that discrimination is justified will report more antifat attitudes, support for obesity public policies, and beliefs in the dangers and controllability of fatness than people exposed to messages of fatness as healthy, uncontrollable, or of discrimination as unacceptable. Furthermore, we expected there would be interactions between controllability and healthiness (e.g., the effect of framing fatness as inherently healthy versus unhealthy on antifat attitudes will be particularly strong when fatness is also presented as under personal control).

Research Question 1. Related to Hypothesis 3, we also tested a key question of interest to public health officials and fat activists alike: Does adding an anti-stigma message to public health crisis and personal responsibility frames reduce antifat stigma?

\section{Overview of Methods: Experiments 1-4}

The experiments were similar to each other in terms of stimuli, dependent measures, and pattern of results, and therefore we describe the methods for all experiments below and organize the results by hypothesis rather than experiment. 


\section{Participants}

Table 1 provides the key demographics for each experiment. University students completed Experiment 1 in exchange for extra course credit. Mechanical Turk workers in the U.S. completed online surveys in Experiments 2 and 3 (ref. 31, 32, 33). Students completed Experiment 4 at the beginning or end of university classes. Of those who completed surveys, we retained only participants who indicated reading the article carefully (Experiments 1-4) and correctly answered three questions about the articles' content (Experiments 2-3): Experiment 1 $(N=511$ out of 657$)$, Experiment $2(N=442$ out of 723$)$, Experiment $3(N=797$ out of 1252$)$, and Experiment $4(N=437$ out of 568). The UCLA IRB granted approval and all participants provided informed consent.

\section{Measures}

Researchers typically ask people about their attitudes toward "obesity" or "the obese," leaving unclear what body types people have in mind when completing the items. To better control this, we presented computer-generated images of men and women from the Body Matrices (ref. 34) that roughly correspond to weights considered Obese I (BMI 30-34.9) and Obese II (BMI 35-39.9) and asked people to keep these images in mind when reporting their attitudes.

Appendix A (Online supplement) provides the full set of measures to which participants responded. Internal consistency was high across all four experiments for all measures (Cronbach's $\alpha$ generally > .70) except for one (workplace discrimination and prejudice), so we averaged the items for each measure. Experiments 1-3 presented all of these measures. Experiment 4 needed to be shorter because it was conducted during classes and therefore only included the Health Risk and Antifat Stigma items. 
Health Risk. We presented 8 computer-generated images of women varying in body fat level taken from the Body Matrices (ref. 34). We featured images of women's bodies because women are subjected to more weight-based discrimination than are men (ref. 35). Participants indicated Yes/No if they believed a woman could be healthy at each of these weights (Appendix $\mathrm{B}$; online supplement). In a separate study, the average BMIs of the women selecting each image as representing their current body were: Image 5 (Overweight: 26), Image 6 (Obese I: 32), Image 7 (Obese II: 39), Image 8 (Obese III: 44).

We also assessed beliefs that fat is unhealthy using a Likert scale measure ( 5 items; $1=$ Strongly Disagree; $9=$ Strongly Agree). This Likert scale was used for the measures described below as well. Cronbach's $\alpha$ s for Experiments $1-4$ were $.89 ; .92 ; .91 ; .87$.

Willpower. We used the Willpower measure to assess beliefs that people can control their weight (3 items; e.g., "Fat people tend to be fat pretty much through their own fault;" as: .70; $.81 ; .77)($ ref. 6$)$

Antifat Stigma. We used three measures of antifat stigma: antifat attitudes ( 5 items (ref. 36); e.g., "On average, fat people are lazier than slender people;” $\alpha$ s: .75; .85; .82; .73), support for workplace prejudice and discrimination toward fat people (3 items; e.g., "Fat people generally aren't as motivated as other slender people in the workplace'” $\alpha \mathrm{s}: .54 ; .71 ; .69 ; .45$ ), and willingness to celebrate body size diversity (3 items; e.g., "People should embrace the idea that "big is beautiful;"' $\alpha \mathrm{s}: .74 ; .85 ; .82 ; .67)$. In contrast to the other measures, higher scores on the body size diversity measure represent more positive attitudes toward fatness.

Policies. We assessed support for all 16 of the policies suggested by Barry and colleagues (ref. 37). These policies can be grouped into three categories. The first is Compensatory policies, which are aimed at helping or protecting citizens (Items 1-6; e.g., "Foods 
with high sugar or fat content should be required to display mandatory warning labels indicating these foods may be addictive;" $\alpha \mathrm{s}: .74 ; .81 ; .80)$. The second is Price-Raising policies, which tend to be more punitive by punishing people financially for being obese or for unhealthy behaviors that can lead to obesity (Items 7-9; "Health insurers should be required to charge higher premiums for policyholders who are obese, allowing them to reduce premiums for everyone else;" as: .66;.71; .74). We also examined the health insurance items separately from the other items. Third, Redistributive policies require taxes to implement (Items 10-16;

"Government funds should be used to establish a national network of obesity treatment programs modeled on treatment for other addictions" $\alpha \mathrm{s}: .83 ; .87 ; .89)$.

\section{Stimuli and Procedure}

We exposed participants to news articles reporting on a fictitious large-scale study, the Harvard Physicians Health Study, purportedly published in the Journal of the American Medical Association. We constructed news articles that were approximately 775 words and two pages single-spaced (Appendices C and D; online supplement). The articles used the same basic text, but varied in whether they presented research evidence and/or quotes from experts indicating that: 1) high body fat as inherently unhealthy or healthy; 2) body fat level is controllable or uncontrollable; 3) stigmatization and weight-based discrimination is acceptable (discriminationjustified) or unacceptable (discrimination-unacceptable). These dimensions were based on previous examinations of the content of common frames in the news media (ref. 2).

In Experiment 1, college students were randomly assigned to one of the two extreme conditions, which we labeled as Fat-Negative (unhealthy + controllable + discriminationjustified; 773 words; Appendix C) or Fat-Positive (healthy + uncontrollable + discriminationunacceptable; 777 words; Appendix D). Experiment 2 tested if Experiment 1's results were 
replicable in a broader adult sample. In Experiment 3, we randomly assigned participants to one of these extreme conditions or to a control condition (no article). In Experiment 4, we used a 2 (Unhealthy / Healthy) X 2 (Controllable / Uncontrollable) X 2 (Discrimination-Justified / Discrimination-Unacceptable) between-subjects experimental design, where we randomly assigned participants to one of the eight possible conditions that could be formed (e.g., unhealthy + uncontrollable + discrimination-unacceptable $)$. This enabled us to test the independent effects of each aspect of the frames and their interactions, compare the extreme conditions, and examine the specific interaction of interest (Research Question 1). Researchers were blinded to group allocation during the experiment.

In order to enhance the mundane realism of the stimuli, in Experiments 1-3 we added text

stating that the articles were published in The New York Times. In Experiment 4, we designed the stimuli so that they appeared as if they were printed off the New York Times website. After reading the news articles, participants reported their attitudes.

\section{Statistical Reporting Standards and Disclosure of Methods}

All statistical tests were two-tailed. We conducted multiple comparisons and therefore report whether results were significant at the $p<.05, .01$, or .001 levels. Following Cumming (ref. 38), we also report effect sizes and 95\% confidence intervals for key results. We report Cohen's $d$ for all mean comparisons. A commonly used guide to interpreting Cohen's $d$ is: close to zero $(d \leq 0.10)$ small $(0.11-0.35)$, medium $(0.36-0.65)$, and large $(66-1.00)$, or very large (>1.00) (ref. 39). Skewness and kurtosis were within commonly accepted ranges for all key variables (all $<|1.1|)$. Standard deviations typically did not differ between groups. When there were small differences (e.g., Levene's test indicating $p<.05$ ), significance was reported using appropriate alternative tests (e.g., unequal variance t-tests). 
EFFECTS OF NEWS ARTICLES 10

It requires approximately 64 participants to detect a positive effect size with $80 \%$ power if the true effect size is $d=.50$ (ref. 40). We therefore elected to collect substantially more than 64 participants per condition in Experiments 1-3. In Experiment 4, where we tested main effects and interactions, we recruited approximately 150 participants per each level of each IV. The number of participants per condition in each experiment was: Experiment $1(N \mathrm{~s}=255,256)$; Experiment $2(N \mathrm{~s}=193,249)$; Experiment $3(N \mathrm{~s}=239,272,286)$; Experiment $4(N \mathrm{~s}=59,45$, $74,53,45,61,41,59)$.

\section{Results}

\section{Hypothesis 1: Fat-Negative versus Fat-Positive (Experiments 1-4).}

In all four experiments, we conducted independent samples $t$-tests comparing the effects of the Fat-Negative versus Fat-Positive articles. We illustrate the pattern of results in the form of Cohen's $d$ for each dependent variable in each experiment (Figure 1). Finally, we conducted Fishers Exact Tests to determine if Article Type had an effect on beliefs that women can be healthy at given weights (Figure 2). We report key statistics (e.g., $p$-values and confidence intervals) in the figures.

Health Risk. Consistent with the hypotheses, Fat-Negative participants reported stronger beliefs that it is unhealthy to be fat compared to Fat-Positive participants, and the effects were quite large (Figure 1). Our key interest was whether the frames would impact people's beliefs that relatively fatter women (images 5-8) can be healthy at their weights. The effects of Article Type were significant in Experiments 1-4 for images 5 (“Overweight”) and 6 (“Obese I") and in Experiments 1-3 for images 7 (“'Obese II”) and 8 (“Obese III”) with Fat-Negative participants being less likely to say they can be healthy at these weights (Figure 2). 
EFFECTS OF NEWS ARTICLES 11

Willpower. Consistent with the hypotheses, Fat-Negative participants reported stronger beliefs that people are fat due to lack of willpower compared to Fat-Positive participants. The effects of the articles were medium-sized (Figure 1).

Antifat Stigma. Consistent with the hypotheses, Fat-Negative participants reported more antifat stigma than Fat-Positive participants in 10 of the 12 possible comparisons. Comparisons were significant in two experiments for antifat attitudes and in all four experiments for workplace prejudice and for willingness to celebrate body size diversity. The effects of the articles were generally small to medium sized (Figure 1).

Policies. Shifting policy attitudes proved more challenging. Only one of six comparisons for compensatory and redistributive policies was significant. Fat-Negative participants did support Price-Raising policies more than Fat-Positive participants in Experiments 1 and 3. As shown in Figure 1, however, the effects were generally small even when significant. Fat-Negative participants reported more support for charging obese people more for insurance in all three studies: (Study 1, $d=.77, p<.001$; Study $2, d=.26, p=.008$; Study $3, d=.34, p<.001)$

\section{Hypothesis 2. Comparisons to Control Condition (Experiment 3).}

In the third experiment, we also conducted one-way ANOVAs with post-hoc LSD tests comparing each experimental condition to the control condition. This allowed us to separately assess the effects of the Fat-Negative and Fat-Positive articles on attitudes relative to Control articles. Figure 3 provides the means, effect sizes (Cohen's $d$ ), $p$-values, and confidence intervals.

Health Risk. Fat-Positive participants reported less belief that fat is unhealthy, and the effect was large. Fat-Negative participants did not report greater support for this belief, although 
EFFECTS OF NEWS ARTICLES 12

this may be due to ceiling effects (mean for Controls exceeded 7.5 out of 9.0, leaving little room for substantial upward movement).

Willpower. Fat-Positive participants reported less belief that fat is controllable, and this effect was medium in size. Fat-Negative did not differ from Controls.

Antifat Stigma. Fat-positive participants reported less antifat stigma on all three measures, and these effects were small to medium. Fat-Negative participants only reported more antifat stigma on the workplace prejudice measure, and the effect was small.

Policies. Fat-Positive participants reported less support for price raising and compensatory policies, but not redistributive policies. Fat-Negative participants did not differ from Controls on any measure. In terms of the insurance item, Fat-Negative participants did not differ from Controls. Fat-Positive participants reported less support for charging obese men and women more for insurance $(d=-.34, p<.001)$.

\section{Hypothesis 3. Effects of Aspects of Frames (Experiment 4).}

The preceding analyses show that it possible to impact attitudes toward fatness when the three key aspects of each frame are joined together. These results leave open the question, however, of how each aspect contributes to the change in attitudes. In the fourth experiment, we tested the effects of each independent aspect of the frames using a 2 (Healthiness: unhealthy / healthy) X 2 (Controllability: controllable / uncontrollable) X 2 (Discrimination: discriminationjustified / discrimination-unacceptable) Between Subjects ANOVA. Figure 4 gives the patterns of means for each dependent variable. Below, we describe the statistically significant main effects and interactions. 
Health Risk. The main effect of Healthiness was the only significant effect $(p<.001)$. People who read articles emphasizing that fatness is unhealthy were more likely than those that did not to report that fatness was a health risk.

Antifat Stigma. Controllability had a significant main effect on antifat attitudes ( $p=$ $.006)$, and a significant interaction with Healthiness $(p=.04)$. Exposure to information that weight is unhealthy only increased antifat attitudes when participants were also exposed to information highlighting the controllability of fatness.

The results for workplace discrimination were similar to those for antifat attitudes (main effect of Controllability ( $p=.04)$, and a significant interaction with Healthiness, $p=.04$ ). However, there was also a main effect of Discrimination on workplace discrimination, with participants reading Discrimination-Unacceptable articles expressing less workplace prejudice $(p$ $=.008)$.

Healthiness had a main effect on support for body size diversity, with people being less willing to celebrate diversity after reading articles emphasizing the unhealthiness of fat ( $p=$ $.003)$.

\section{Research Question 1. Effectiveness of Adding Discrimination-Unacceptable Messages to Public Health Crisis - Personal Responsibility Frames (Experiment 4).}

Of particular interest is whether attaching a message about the unacceptability of weightbased discrimination to the standard public health framing can protect against the antifat bias that this frame might otherwise generate. We compared whether people who read the unhealthy + controllable + discrimination-unacceptable article reported less antifat stigma than people who read the unhealthy + controllable + discrimination-justified article. They did report less support 
EFFECTS OF NEWS ARTICLES 14

for workplace discrimination $(d=.56, p=.008)$. However, they did not report fewer antifat attitudes $(d=.04 p=.85)$ or more celebration of body size diversity $(d=-.17, p=.40)$.

\section{Discussion}

\section{Key Findings and Implications}

Consistent with Hypothesis 1, exposure to the Fat-Negative versus Fat-Positive frames reliably shifted beliefs that fat is inherently unhealthy, beliefs that fatness is controllable, and the expression of antifat stigma. In Experiment 3, the Fat-Positive-but not the Fat-Negativeframes shifted these attitudes consistently compared to the Control conditions. It may be that people's preexisting negative views of fat means that only exposure to extensive additional negative information can increase these negative attitudes.

In contrast, it was more difficult to shift policy attitudes. Exposure to scientific claims that fatness poses health risks had little or no impact on policy support, casting doubt on the idea that disseminating such information increases public support for obesity policies. Gollust et al. (ref. 41) suggest that other appeals - such as highlighting the impact of health issues on military readiness - may be more effective. The exception is that, compared to both Controls and FatNegative frames, Fat-Positive frames reduced support for charging obese men and women more for health insurance. These results suggest that more fat-positive news framing could help erode support for such surcharges, which employers are permitted to charge under the Patient Protection and Affordable Care Act. Doing away with such surcharges would have positive health and financial implications for those categorized as obese.

Consistent with Hypothesis 3 and the Justification-Suppression model of prejudice (ref. 4), people expressed more antifat attitudes and workplace prejudice after reading information suggesting that weight is controllable, especially when they also read that being fat was 
unhealthy. In contrast to our hypotheses, only healthiness of fatness - and not controllabilityreduced willingness to celebrate body size diversity.

Findings were mixed for Research Question 1. Adding discrimination-unacceptable messages to articles portraying fatness as unhealthy and controllable reduced the expression of workplace prejudice relative to the Fat-Negative article. It did not, however, reduce antifat attitudes or increase willingness to celebrate body size diversity. Effects may have been limited to the workplace discrimination outcome measure because the anti-discrimination text focused primarily on discrimination prevention in the workplace. Considering the harmful effects of stigma, future work should examine whether broader anti-weight-stigma messages can alleviate more general forms of antifat stigma.

\section{Limitations and Strengths}

The items in the workplace prejudice and discrimination scale were only modestly intercorrelated, raising concerns about the validity of findings with that scale. We used controlled news articles that appeared to be taken from an influential newspaper, and our results suggest they were sufficiently believable to shift attitudes. Future research could examine whether stronger belief in the validity of the studies mediates effects. Constructed news articles allowed us to assess the independent effects of each frame. Our samples were large, providing more precise estimates of effect size, and we replicated our findings across multiple experiments. Notably, we showed participants computer-generated images of fat men and women to assess their beliefs about weight. Without the use of such images, asking people to envision a person who is "obese" or "fat" may lead them to imagine people at the extreme end of the weight spectrum.

\section{Conclusion}


In four experiments, we examined how exposure to specific fat frames - and specific aspects of these frames - affected attitudes about health, support for health policy, and antifat bias and discrimination. Reading that weight-based discrimination is unacceptable, within a context of an article about obesity as a public health crisis brought on by bad personal choices, did not reduce the expression of weight-based stigma. However, pairing the message of the unacceptability of weight-based discrimination with the concept of health at every size and uncontrollability of body weight did reduce the expression of weight-based stigma. These results suggest that current high levels of reporting on obesity as a public health crisis due to lack of personal responsibility undermine efforts to reduce antifat stigma and its negative associated health implications. Rather, to reduce antifat stigma, more public discussion is needed on health at every size and the factors beyond personal control that shape body weight.

Supplementary information is available at IJO's website. 


\section{Sources Cited}

1. Saguy AC, and Almeling R. Fat in the fire? Science, the news media, and the 'obesity epidemic'. Sociological Forum. 2008;23:53-83.

2. Saguy AC. What's Wrong with Fat? New York: Oxford University Press; 2013.

3. Saguy AC, Gruys K, and Gong S. Social problem construction and national context: News reporting on 'overweight' and 'obesity' in the U.S. and France. Social Problems. 2010;57:586-610.

4. Crandall CS, and Eshleman A. A justification-suppression model of the expression and experience of predudice. Psychological Bulletin. 2003;129:414-46.

5. Crandall CS, D’Anello S, Sakalli N, Lazarus E, Nejtardt GW, and Feather NT. An attribution-value model of prejudice: Anti-fat attitudes in six nations. Personality and Social Psychology Bulletin. 2001;27:30-7.

6. Crandall CS. Prejudice against fat people: Ideology and self-interest. Journal of Personality and Social Psychology. 1994;66:882-94.

7. Puhl RM, and Latner JD. Stigma, obesity, and the health of the nation's children. Psychological Bulletin. 2007;133:557-80.

8. Muennig P, and Bench KK. Obesity-associated stigma and physiological markers of stress: Evidence from the Dominican Republic. Stress and Health. 2008;25:241-6.

9. Puhl RM, and Heuer CA. Obesity stigma: Important considerations for public health. American Journal of Public Health. 2010;100:1019-28.

10. Pace G. CBSNews. Columbia, S.C.; 2006. 
11. Saguy AC, and Gruys K. Morality and health: News media constructions of overweight and eating disorders. Social Problems. 2010;57:231-50.

12. Heuer CA, McClure KJ, and Puhl RM. Obesity stigma in online news: a visual content analysis. Journal of health communication. 2011;16:976-87.

13. Puhl RM, Peterson JL, DePierre JA, and Joerg L. Headless, hungry, and unhealthy: A video content analysis of obese persons portrayed in online news. Journal of Health Communication. 2013;18:686-702.

14. Bacon L. Health at Every Size: The Surprising Truth about Your Weight. Dallas: BenBella Books; 2010.

15. Mann T, Tomiyama AJ, Westling E, Lew A-M, Samuels B, and Chatman J. Medicare's search for effective obesity treatments: Diets are not the answer. American Psychologist. 2007;62:220-33.

16. Campos P, Saguy AC, Ernsberger P, Oliver E, and Gaesser G. The epidemiology of overweight and obesity: Public health crisis or moral panic? International Journal of Epidemiology. 2006;35:55-60.

17. Dodge R. Knight Ridder/Tribune News Service. 2005.

18. Marchione M. The Associated Press. 2005.

19. Cooper C. Fat and Proud: The Politics of Size. London: Women's Press; 1998.

20. Wann M. FAT!SO?: Because You Don't Have to Apologize for Your Size. Berkeley, CA: Ten Speed Press; 1999.

21. Rothblum ED, and Solovay S eds. The Fat Studies Reader. New York, NY: New York University Press; 2009. 
22. Harding K, and Kirby M. Lessons from the Fat-O-Sphere: Quit Dieting and Declare a Truce with Your Body. New York, NY: Pengine Group; 2009.

23. Saguy AC, and Riley KW. Weighing both sides: Morality, mortality and framing contests over obesity. Journal of Health Politics, Policy, and Law. 2005;30:869-921.

24. Daníelsdóttira S, O'Brien KS, and Ciao A. Anti-fat prejudice reduction: A review of published studies. Obesity Facts. 2010;3:47-58.

25. Saguy AC, Frederick D, and Gruys K. Reporting risk, producing prejudice: How news reporting on obesity shapes attitudes about health risk, policy, and prejudice. Social Science \& Medicine. 2014;111:125-33.

26. McClure KJ, Puhl RM, and Heuer CA. Obesity in the news: do photographic images of obese persons influence antifat attitudes? Journal of Health Communication. 2011;16:359-71.

27. Pearl RL, Puhl RM, and Brownell KD. Positive media portrayals of obese persons: Impact on attitudes and image preferences. Health Psychology. 2012;31:821-9.

28. Puhl RM, Heuer CA, and Sarda V. Framing messages about weight discrimination: Impact on public support for legislation. International Journal of Obesity. 2011;35:86372.

29. Brochu PM, Pearl RL, Puhl RM, and D. BK. Do media portrayals of obesity influence support for weight-related medical policy? Health Psychology 2014;33:197-200.

30. Gollust SE, Niederdeppe J, and Barry CL. Framing the consequences of childhood obesity to increase public support for obesity prevention policy. American Journal of Public Health. 2013:e1-e7. 
31. Berinsky AJ, Huber GA, and Lenz GS. Evaluating online labor markets for experimental research: Amazon.com's Mechanical Turk. Political Analysis. 2012;20:351-68.

32. Buhrmester M, Kwang T, and Gosling SD. Amazon's Mechanical Turk a new source of inexpensive, yet high-quality, data? Perspectives on Psychological Science. 2011;6:3-5.

33. Paolacci G, Chandler J, and Ipeirotis PG. Running experiments on Amazon Mechanical Turk. Judgment and Decision Making. 2010;5:411-9.

34. Gray PB, and Frederick DA. Body image and body type preferences in St. Kitts, Caribbean: A cross-cultural comparison with U.S. samples regarding attitudes towards muscularity, body fat, and breast size. Evolutionary Psychology. 2012;10:631-55.

35. Puhl RM, Andreyeva T, and Brownell KD. Perceptions of weight discrimination: Prevalence and comparison to race and gender discrimination in America. International Journal of Obesity. 2008;32:992-1000.

36. Morrison TG, and O'Connor WE. Psychometric properties of a scale measuring negative attitudes toward overweight individuals. Journal of Social Psychology. 1999;139:436-45.

37. Barry CL, Brescoll VL, Brownell KD, and Schlesinger M. Obesity metaphors: How beliefs about the causes of obesity affect support for public policy. Milbank Quarterly. 2009;87:7-47.

38. Cumming G. The new statistics: Why and how. Psychological Science. 2014;25:7-29.

39. Hyde JS. The gender similarities hypothesis. American Psychologist. 2005;60:581-92.

40. Simonsohn U. Data Colada. 2014.

41. Gollust SE, Eboh I, and Barry CL. Picturing obesity: Analyzing the social epidemiology of obesity conveyed through US news media images. Social Science \& Medicine. 2012;74:1544-51. 
EFFECTS OF NEWS ARTICLES 21

\section{Figure Legends}

Figure 1. Framing Effects on Attitudes towards Fat for Full Sample, Experiments 1-4.

Figure 1 Note. Positive effect sizes indicate that Fat-Negative participants reported stronger agreement with the statements than Fat-Positive. Error bars represent $95 \%$ confidence intervals.

Figure 2. Framing Effects on Beliefs Woman Can Be Healthy at Different Weights, Experiments $1-4$.

Figure 2 Note. $* p<.05, * * p<.01, * * * p<.001$. Asterisks indicate that the condition differed significantly from the condition immediately to the right (e.g., $93 \%$ vs. 87\%, $p<.05$ ).

Figure 3. Framing Effects on Beliefs about Health Risks and Antifat Stigma, Experiment 3.

Figure 3 Note. $* p<.05, * * p<.01, * * * p<.001$. Error bars represent $95 \%$ confidence intervals. Values above bars represent Cohen's $d$ for comparisons of each column to the Control condition (e.g., Fat-Positive participants were less likely than Control participants to believe that fat is unhealthy, $d=-.76$ ).

Figure 4a-d. Framing Effects on Beliefs about Health Risks and Antifat Stigma, Experiment 4.

Figure 4 Note. Error bars represent $95 \%$ confidence intervals. 
Table 1. Sample Characteristics

\begin{tabular}{|c|c|c|c|c|}
\hline & \multicolumn{4}{|c|}{ Experiment } \\
\hline & 1 & 2 & 3 & 4 \\
\hline Sample Type & $\begin{array}{l}\text { Univ- } \\
\text { ersity }\end{array}$ & $\begin{array}{l}\text { Mech } \\
\text { Turk }\end{array}$ & $\begin{array}{l}\text { Mech. } \\
\text { Turk }\end{array}$ & $\begin{array}{l}\text { Univ- } \\
\text { ersity }\end{array}$ \\
\hline $\begin{array}{l}\text { Number Experimental } \\
\text { Conditions }\end{array}$ & 2 & 2 & 3 & 8 \\
\hline $\begin{array}{l}\quad \text { Sample Size } \\
\text { Total }(N) \\
\text { Women }(N) \\
\text { Men }(N)\end{array}$ & $\begin{array}{l}511 \\
376 \\
135\end{array}$ & $\begin{array}{l}442 \\
195 \\
247\end{array}$ & $\begin{array}{l}797 \\
437 \\
360\end{array}$ & $\begin{array}{l}437 \\
332 \\
115\end{array}$ \\
\hline $\begin{array}{ll} & \text { Age } \\
M & \\
(S D) & \end{array}$ & $\begin{array}{l}21.2 \\
(2.7)\end{array}$ & $\begin{array}{c}35.4 \\
(11.7)\end{array}$ & $\begin{array}{l}37.2 \\
(12.7)\end{array}$ & $\begin{array}{l}21.1 \\
(2.3)\end{array}$ \\
\hline $\begin{array}{l}\quad \text { Ethnicity } \\
\text { White }(\%) \\
\text { Asian }(\%) \\
\text { Hispanic }(\%) \\
\text { Black (\%) } \\
\text { Other }(\%)\end{array}$ & $\begin{array}{c}31 \\
43 \\
6 \\
1 \\
19\end{array}$ & $\begin{array}{l}75 \\
8 \\
6 \\
7 \\
4\end{array}$ & $\begin{array}{c}81 \\
5 \\
4 \\
6 \\
4\end{array}$ & $\begin{array}{c}57 \\
24 \\
9 \\
2 \\
8\end{array}$ \\
\hline $\begin{array}{l}M \\
(S D)\end{array}$ & $\begin{array}{l}22.9 \\
(4.2)\end{array}$ & $\begin{array}{l}26.0 \\
(6.4)\end{array}$ & $\begin{array}{l}24.8 \\
(5.9)\end{array}$ & $\begin{array}{l}22.5 \\
(3.8)\end{array}$ \\
\hline
\end{tabular}




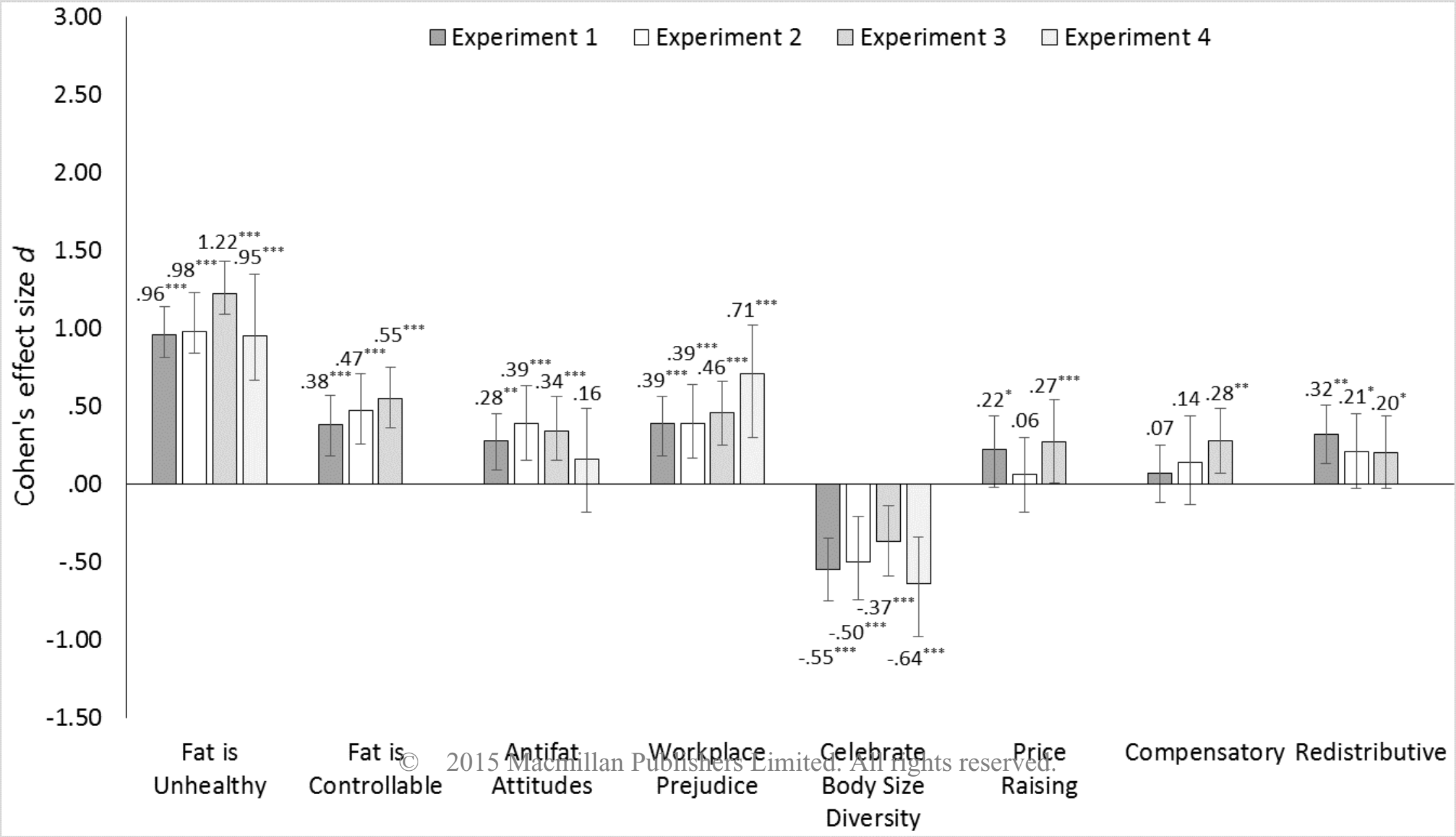




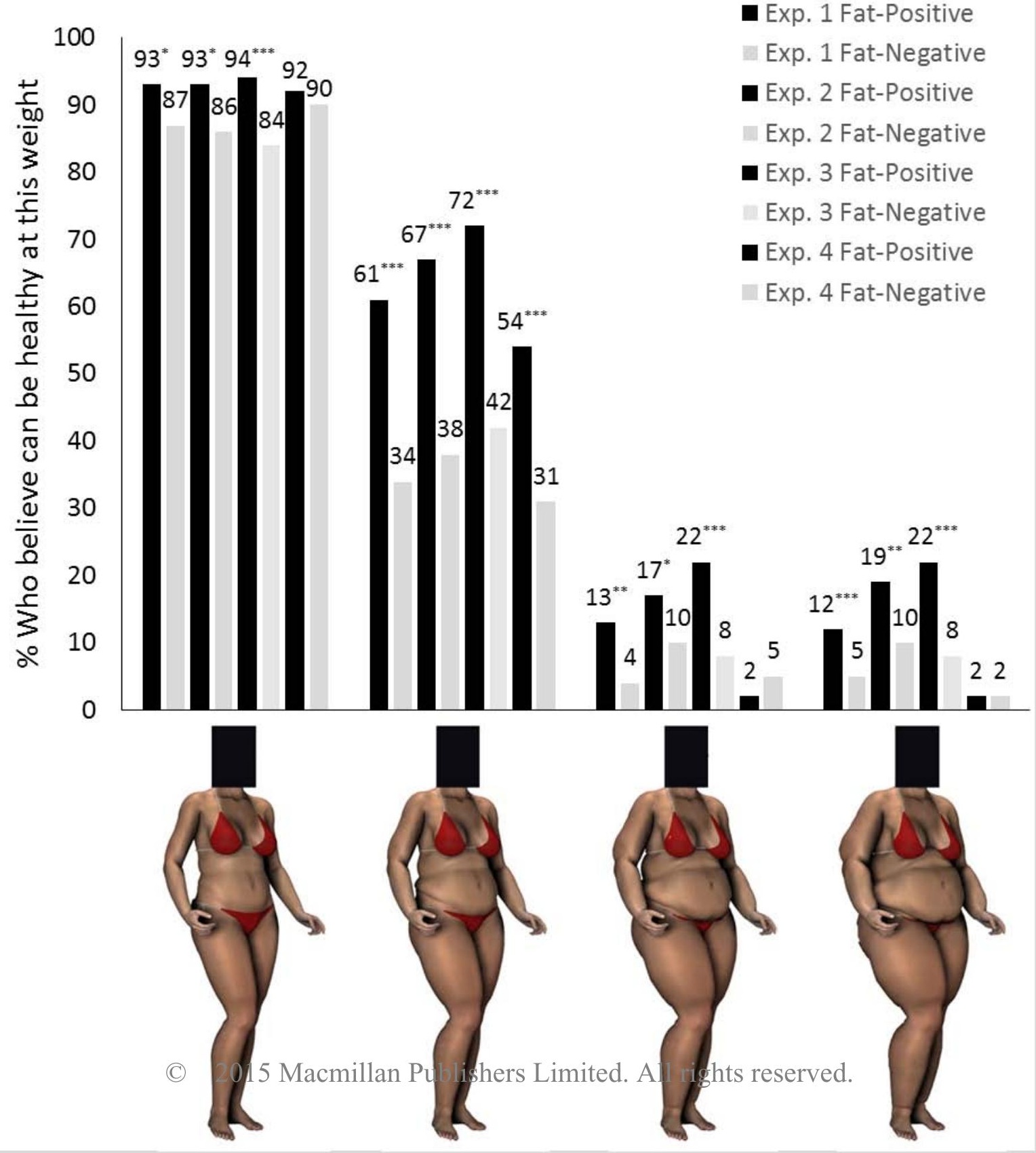



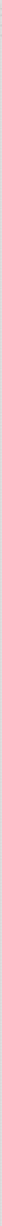


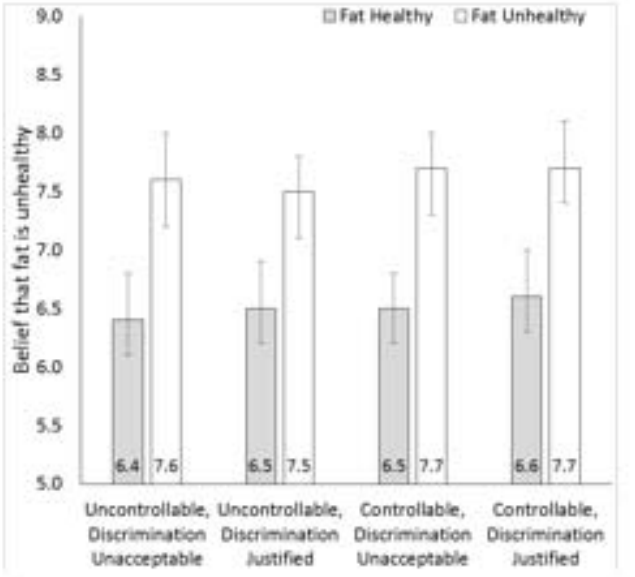

4c) Workplace Discrimination
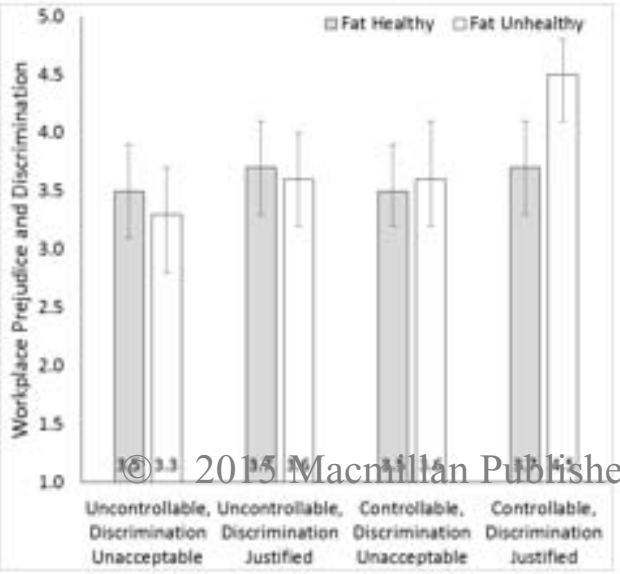

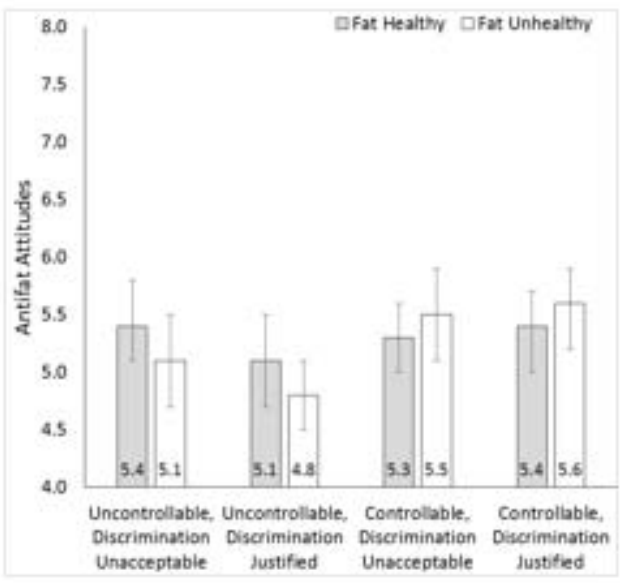

4d) Celebrate Body Size Diversity

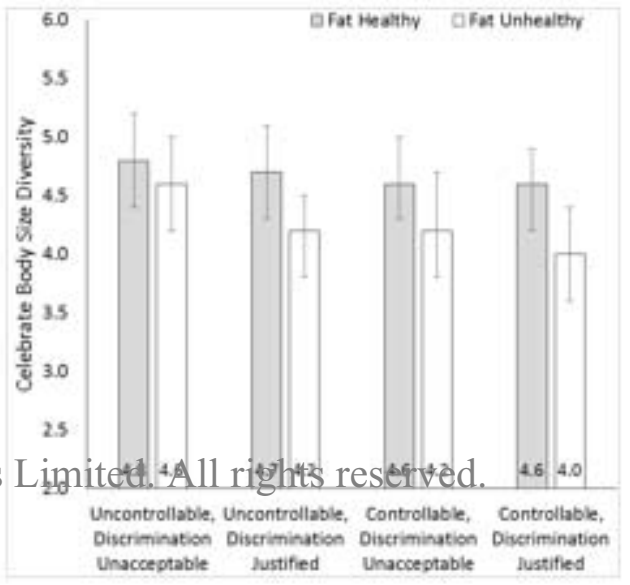

